\title{
La violence urbaine à Johannesburg. Entre réalité et prétexte
}

\section{Philippe Guillaume, Choisy-le-Roi}

\section{Introduction}

C'est devenu une banalité de présenter Johannesburg comme une city of crime. Les agences de voyage, par exemple, nombreuses à proposer des circuits en Afrique du Sud (montagnes et océans du Cap, route des vins, réserves animalières du Kruger), encadrent très soigneusement leurs clients dès la sortie de l'aéroport. Si une étape est prévue dans la province du Gauteng, elle se fera à Tshwane (nouveau nom de Pretoria). Les guides touristiques ont très nettement changé de ton ces dernières années: en 1995, le Petit Futé se contentait de quelques mises en garde contre l'insécurité. En 1997, il signalait que dans le centre de la ville, «il fallait se balader à poil pour être sûr de ne pas se faire attaquer». En 2000, enfin, l'auteur avouait qu'il aurait des problèmes de conscience à recommander la visite de la ville à des touristes. L'évolution du discours est nette. La ville a déjà fait l'objet de mises au ban internationales et semi-officielles. Certains pays, par le biais de leur consulat, déconseillent très vivement à leurs ressortissants de visiter la ville: c'est le cas de l'Allemagne, par exemple, depuis l'agression et le viol collectif d'un groupe d'adolescentes par un gang armé, dans une auberge de jeunesse à l'été 2000. La presse étrangère joue évidemment son rôle dans la diffusion de ces drames. Un magazine à grande diffusion tel que GÉo présentait Johannesburg comme un «mauvais rêve», dans un reportage d'avril 1999. Le sommaire était sans équivoque: il s'agissait de rendre compte d'une «cité exsangue qu'abandonnent les grandes entreprises et que fuient ses habitants les moins démunis».

Dans l'article suivant, DidIER LAINÉ signalait (à tort, d'ailleurs) ce qui lui semblait être la première forme d'égalité des Blancs et des Noirs après l'apartheid, celle qui les unissait devant la Mort. Le pourtant estimable MONDE DES DÉBATS surenchérissait en mai 1999, où un article de PHILIPPE TRÉTIACK présentait la métropole comme «un radieux coupe-gorge» avec un intertitre «36 fois par jour», relatif au nombre de vols de voiture avec violence. La photographie choisie est celle d'un chauffeur de taxi gisant dans une rue du centreville, une balle dans la tête. Certains passages sont lyriques: ce qui est décrit n'est seulement que «le sommet d'un iceberg de sang frais».

La presse sud-africaine n'est pas en reste. Le quotidien le plus diffusé à Johannesburg, The Star, propose chaque jour en page deux le compte rendu des crimes et faits divers de la veille. Le format est standardisé: le nom du quartier figure en gras, suivi du nom des victimes, des circonstances de l'agression et du détail des blessures ou impacts de balle. Il est évident que cette rubrique influence nettement les perceptions qu'ont les citadins de tel ou tel quartier de la ville: les lieux sont rapidement affublés d'un «niveau de fréquentabilité» et cette démarche, plus ou moins consciente, entraîne à son tour des modifications des itinéraires et des destinations, dans un processus somme toute assez classique de construction de cartes mentales. Comme si cela ne suffisait pas, THE STAR a régulièrement publié des cartes mensuelles faisant le bilan des attaques de banques et de bureaux de poste (quartier par quartier, avec un niveau de détail en fonction des succursales) ainsi que des hijacks, ces vols de voiture en circulation et à main armée. Lorsque l'on se rend à sa banque, par exemple, il est donc possible de faire des suppositions concernant à la fois le quartier où se trouve l'agence la plus sûre et l'itinéraire le moins risqué pour y aller. Cela peut rendre le quotidien moins routinier. La violence urbaine est donc omniprésente dans les informations et dans les discussions entre les individus: il y a toujours une anecdote horrible à se raconter. Elle figure également comme acteur principal de nombreuses créations culturelles contemporaines et ce, quel qu'en soit le support: musique, pièce de théâtre, films ou séries télévisées.

L'un des premiers problèmes posés par cette violence est qu'elle participe à la négation même de l'idée de ville, à la possibilité d'un projet commun réunissant des citadins se revendiquant encore comme citoyens. Dans les stratégies résidentielles (quitter un logement, en choisir un nouveau, rêver d'un quartier où l'on aimerait habiter), la question de la sécurité prime sur toutes les autres considérations, y compris celles liées à l'accessibilité, aux services, à l'éducation ou encore aux commerces. Pourtant, dire que la ville sud-africaine n'est pas urbaine n'a rien d'original: JACQUES LÉvY (1999) la définissait il y a plusieurs années comme «un crime contre l'urbanité». Mais c'est de la ville post-apartheid dont on parle ici, celle-là même qui est porteuse d'un projet, et non des moindres.

Dans le débat qui a accompagné la transition politique et l'avènement de la démocratie, dans les expertises sud-africaines et internationales, (Banque Mondiale, Urban Foundation, National Housing Forum) ou encore dans le Programme de Reconstruction et de Développement (RDP) qui a porté l'African Natio- 
nal Congress (ANC) à la victoire, les recommandations sont identiques. Parmi les axes à suivre, qui permettront de s'affranchir définitivement de l'héritage inique de l'apartheid et de construire une nouvelle société libre, démocratique, non raciale et égalitaire, se détache un projet extrêmement fort donnant une place de choix à la ville. C'est en effet en partie par la reconnaissance de la citadinité que se fera l'accès à la citoyenneté. Pour cela, les villes post-apartheid devront privilégier le contact, la densité, la mixité, la diversité et l'accessibilité, en un mot, l'urbanité. Beau programme révolutionnaire.

Or, les barricades ne sont pas là où on les imagine. Il est peu réducteur de dire que les premières élections démocratiques et non raciales se sont accompagnées d'un net sentiment d'une très forte progression de l'insécurité. Les villes sud-africaines, et particulièrement Johannesburg, semblent être atteintes par une violence sourde et aveugle, frappant à la fois de manière aléatoire et quasi systématique. L'établissement de causalités directes entre la fin de l'apartheid et l'irruption de la violence sur la scène publique serait là, par contre, parfaitement réducteur. Toujours est-il que les quartiers se mettant à l'écart de la vie publique sont de plus en plus nombreux: les rues se ferment, des gardiens armés surveillent des points d'accès de plus en plus réduits et, dans les gigantesques banlieues résidentielles de la métropole, cette dynamique est devenue la norme. La forte demande fait monter les prix; les résidents, heureux, tiennent tous le même discours: ils sont satisfaits d'être à l'abri de l'insécurité. La ville, pendant ce temps, se modèle selon des forces éloignées de ce qui était prôné par le RDP: l'espace public, comme pratique et comme concept, tend à disparaître et il y a atteinte à la démocratie, du moins à celle qui était programmée. Le parallèle entre la fin de l'apartheid, la montée de la violence et l'évolution vers une ville sécuritaire est simple, facile, presque évident. Et si cette violence, bien que réelle, était une mauvaise excuse?

Cet article n'a qu'une ambition: tenter d'analyser les phénomènes de violence urbaine en Afrique du Sud, à Johannesburg en particulier, en articulant la violence réelle et la production des discours sur la ville, en observant la mutation discrète mais sournoise des rapports à la «race», et, enfin, en la plaçant comme enjeu fondamental d'une société en transition.

\section{L'apologie du rempart}

Les concepts de distance et de contrôle sont essentiels à la compréhension de la ville d'apartheid. La société sud-africaine, en effet, ne s'est jamais pensée autrement que par la distance: on sait que dans les années qui ont suivi l'arrivée des Afrikaners au Cap, en 1652, des mesures visaient déjà à séparer dans l'espace de la colonie les Européens des indigènes, les civilisés des non civilisés. Dans le courant du XIXe siècle, des lois empêchaient ça et là les Africains de résider dans les rues principales des villes. Au début du XXe siècle, l'esprit du système évolue: une épidémie de peste à Johannesburg touche plusieurs milliers de personnes dans des quartiers populaires au peuplement assez mixte. Comme le disait Michel Foucault (1975) dans "Surveiller et punir», les dirigeants rêvent souvent de la peste pour pouvoir faire appliquer les lois. L'occasion était donc trop belle: le quartier est détruit, les non-Blancs sont déplacés à vingt kilomètres du centre de Johannesburg et les Afrikaners se trouvent de fait protégés d'influences jugées néfastes. Belle métaphore sanitaire. L'apartheid, consécutif à la victoire du National Party aux élections de 1948, ne fera ensuite qu'uniformiser des mesures finalement anciennes. Les bantoustans se créent dans les campagnes désolées et chaque ville se dote d'un ou de plusieurs townships.

À Johannesburg comme ailleurs, tous les quartiers mixtes et péricentraux seront détruits; le déjà mythique Sophiatown ne sera pas épargné par le mouvement. Le Central Business District (CBD) reste, pendant l'apartheid, un espace professionnel et commercial fréquenté par toutes les «races», mais la mixité n'est de mise que pendant la journée. Le soir, les «non-Blancs» doivent franchir la ligne des terrils bordant le sud du CBD et qui agit comme une véritable frontière symbolique: c'est elle qui assure les richesses de la ville et du pays, c'est elle que doivent dépasser la majorité des exclus de la citoyenneté pour atteindre leur township. Soweto se trouve donc à vingt kilomètres au sud-ouest de Johannesburg, tout comme Eldorado Park, le township métis. Les Indiens, placés de force à Lenasia, doivent aller encore plus au sud. Les Blancs, eux, disposent de la moitié nord de la métropole, d'abord dans des quartiers d'immeubles tels qu'Hillbrow ou Berea, ensuite dans des lotissements pavillonnaires comme Rosebank ou Sandton.

Mais si l'espace construit peut être déjà considéré comme la caricature de la ville de la ségrégation, la relative proximité euclidienne ne peut masquer une faille cognitive. Car si l'on parle de Johannesburg comme une ville, cette ville recouvre pourtant deux mondes, voire deux univers. Il n'y a pas de plus belle illustration de cette rupture que celle proposée par ANDRÉ BRINK (1979) dans son roman «Rumeurs de pluie», mettant en scène Martin Mynhardt, brillant et séduisant Afrikaner, propriétaire de mines, secondé par Charlie Mofokeng, habile négociateur dans les conflits avec les mineurs dont il parle la langue. Un jour, de retour vers Johannesburg :

\footnotetext{
- Charlie: «Tu n’as jamais mis les pieds à Soweto ?»

- Martin: «Bien sûr que non, qu'est-ce que j'irais y faire ?»
} 
Pour ce quadragénaire blanc, mettre les pieds dans un township est une hérésie. C'est moins une question de métrique que d'espace biographique: le Group Areas Act a posé des remparts. Chaque individu, en fonction de sa «race», n'a accès qu'à une fraction de la ville. Et si les Africains doivent parfois, de facto, fréquenter des quartiers «blancs», l'inverse n'est pas envisageable. Alors Charlie force le destin et Martin se retrouve, plongé dans un abîme de perplexité, dans un univers qui n'est pas le sien:

«Je n'y avais jamais été (et n'y suis pas retourné depuis). Et mes souvenirs sont confus - non pas à cause de l'accumulation d'impressions, mais parce que j'étais extrêmement furieux contre Charlie. Et j'avais peur. Je dois l'admettre. C'est peut-être la seule expérience de ma vie d'adulte qui m'ait vraiment fait peur; alors que je n'arrivais pas à définir la raison de cette peur. C'est ce qu'il y a de pire. Rien n'est arrivé et tout pouvait arriver, à tout instant. Si nous avions été arrêtés par la police? Un homme dans ma situation, l'incident aurait immédiatement pris une coloration politique. Que faisait Martin Mynhardt à Soweto? Ils auraient pu me liquider à cause de ça, s'ils en avaient eu envie: en Afrique du Sud, d'autres personnes avaient été liquidées pour des raisons moins graves.»

La peur: sentiment étrange et confus. L'entrée de MarTin MynhardT dans Soweto est un véritable choc: il reçoit brutalement la confirmation qu'existent dans ce pays des mondes parallèles, des pratiques sociales, des paysages différents construits et imposés à des populations conçues comme différentes. MarTiN en ressortira troublé à jamais: il n'est plus possible désormais de se persuader qu'il «ne savait pas», de faire semblant d'y croire. C'est peut-être cela le plus frappant: la capacité des Blancs sud-africains à se cacher la vérité, à se persuader que tout va bien, que tout fonctionne normalement.

Pendant les années les plus dures de l'apartheid, les Blancs sud-africains sont à l'abri de toute forme de violence. Le pays est riche, l'économie enregistre de forts taux de croissance, le système d'apartheid est extrêmement protecteur à l'égard des Blancs. Quels que soient leurs niveaux de qualifications et de compétences, la simple couleur de peau leur garantit un niveau de vie toujours décent, souvent plus que confortable. L'état policier assure la sécurité et la faiblesse des contestations.

L'Afrique du Sud, pourtant, est un pays violent. Simplement la violence se produit de l'autre côté des remparts et n'est médiatisée que lors de circonstances exceptionnelles: les émeutes de Sharpeville en 1960. A l'origine une campagne de résistance passive à l'encontre des pass; la police, pourtant, a ouvert le feu sur les manifestants, faisant 67 morts et 156 blessés, la plu- part ayant reçu des balles dans le dos. Ou de Soweto en 1976, par exemple. A l'origine, un mouvement de résistance pacifique contre la décision d'instaurer l'enseignement en afrikaans. Là encore, les réactions des autorités semblent démesurées par rapport à la teneur des revendications et l'état d'urgence sera imposé. Hector Petersen, un écolier tué par la police, sera la première victime et l'emblème de cette lutte. Mais le mouvement s'étendra, porté par des adolescents prêts à se sacrifier pour la cause. La désorganisation touchera l'ensemble du pays pendant plusieurs mois et l'image de l'Afrique du Sud sera à jamais altérée sur la scène internationale. La violence quotidienne, elle, passe généralement sous silence. Elle est pourtant loin d'être négligeable.

$\mathrm{Si}$, de nos jours, il est possible de disserter sur l'aspect déshumanisant des townships, leur manque d'harmonie et de diversité, leur faible potentiel ou encore la nécessité d'opérations massives de développement, il est plus difficile de se rendre compte de l'humiliation constante qu'a été l'apartheid aux yeux des populations dites «de couleur».

Une éducation au rabais, des mobilités contrôlées avec les pass, une citadinité contrainte (propriété foncière interdite et menace permanente d'exclusion dans les bantoustans, impossibilité de choisir sa maison ou son quartier), des couvre-feux, des salaires de misère, l'interdiction de se réunir ou d'exprimer des opinions politiques, la pression constante des autorités (contrôles, raids policiers), des emprisonnements massifs et des citoyens bannis: tel est l'univers des townships. La répression produira des générations de personnes résignées, acceptant leur sort en silence. D'autres, par contre, ne trouvent dans la violence que le seul moyen d'expression qui reste à leur disposition. MARCANTOINE DE Montclos (1997) note que la violence à l'intérieur de Soweto a triplé de 1960 à 1977. La pauvreté, les privations et frustrations, la répression, enfin, en sont les principales causes. Clive Glaser (1988) note que la fin des années 1960 marque une croissance nette dans l'organisation des gangs à Soweto: ils n'étaient qu'une dizaine vers 1965 , ils étaient plus de cinquante dix ans plus tard, certains étant capables de mobiliser plus de cent personnes. L'augmentation de leur audience est probablement due, selon Clive GlASER, au taux de chômage très élevé ainsi qu'à la part de la jeunesse qui n'est pas scolarisée.

Dans les années 1970, les gangs s'éloignent définitivement de l'image "glamour» qu'ils véhiculaient dans les années 1950, à l'époque de la culture de Sophiatown, où la ville sud-africaine était encore véritablement urbaine. La violence, du coup, devient plus présente. De plus en plus de gangs puisent leur légitimité là où ils le peuvent: les comrades, activistes politiques 
et les tsotsis, figures anciennes des gangsters, fusionnent en comtsotsis. Ces bandes se donnent donc un rôle dans la libération du pays par la violence: les bâtiments publics sont saccagés, les "collaborateurs» ou «traîtres» sont brûlés vifs, entourés par un pneu imbibé d'essence dans le célèbre «supplice du collier». En plus de la violence classique de prédation économique, cette violence socio-politique donnera lieu à de nombreux débordements et règlements de compte. Tout cela, pourtant, reste étranger à l'univers des Blancs.

Dans un roman, «l'Age de fer», Michael Coetzee (1992) met en scène Elisabeth Curren, sud-africaine blanche de classe moyenne, personnage banal et «moyen» dans cette société. Un jour, accompagnant exceptionnellement sa domestique dans son township, elle est témoin de scènes de violence, se trouvant

«à l'arrière d'une foule de plusieurs centaines de personnes qui contemplait un spectacle de dévastation, des baraques brûlées dont les débris rougeoyaient encore, des baraques en flamme d'où montait une fumée noire.»

Se sentant extrêmement mal à l'aise, elle s'adresse à la seule personne qu'elle connaisse dans ce township, M. Thabane, le cousin de sa maid:

"Il faut que je rentre vite chez moi.»

"Vous en avez assez vu?», demanda M. Thabane, l'air plus distant qu'auparavant.

«Oui, je ne suis pas venue pour voir, je suis venue pour chercher Bheki.»

«Et vous voulez rentrer chez vous?»

«Oui, j’ai mal, je suis épuisée.»

"Vous voulez rentrer chez vous... Et les gens qui vivent ici? Quand ils veulent rentrer chez eux, c'est ici qu'ils doivent aller. Qu'en pensez-vous?»

Nous étions debout sous la pluie, au milieu du chemin, face à face. Des passants se sont arrêtés à leur tour, m'examinant avec curiosité. Mes affaires les regardaient, elles regardaient tout le monde.

«Je n'ai rien à répondre. C'est terrible.»

"Terrible, ça ne suffit pas. C'est un crime. Quand vous voyez un crime se commettre sous vos yeux, qu'est-ce que vous dites? Est-ce que vous dites: cj'en ai assez vu, je ne suis pas venue pour voir, je veux rentrer chez moi? »"

M. Thabane accompagnera finalement Elisabeth Curren à sa voiture.

«Je vous demande pardon, je ne suis pas sûre de pouvoir retrouver mon chemin.»

"Continuez jusqu'à la route, tournez à droite, suivez les signaux.»

«Oui, mais quels signaux?»

«Les signaux qui indiquent la civilisation.»

L'expérience est définitivement traumatisante. Elisabeth Curren en tirera une grande honte:

"Chez moi, dans ma maison tranquille, dans mon lit de sommeils enfantins. Ai-je jamais été pleinement éveillée?»
C'est probablement la sécurité qui était le plus puissant des privilèges des Blancs: dans cette société extrêmement inégalitaire, policière et répressive, où la violence est sous-jacente, seuls les Blancs pouvaient vivre dans le confort et la tranquillité, comptant sur l'État et le système pour préserver les intérêts de leur famille et de leur communauté. Il en allait tout autrement dans les townships. La fin de l'apartheid a eu l'effet d'une véritable démolition des remparts: en rendant aux «non-Blancs» l'accès à la totalité de l'espace du pays, l'Afrique du Sud s'est dotée d'une nouvelle mémoire collective. La paix, le pardon, la volonté de réconciliation et de reconstruction sont désormais communs à tous. Les défis, le chômage, la violence également. Dans le contexte propre à ce pays, la montée des préoccupations sécuritaires consécutive à l'abolition de l'apartheid n'apparaît donc pas comme irrationnelle et constitue un axe structurant de l'évolution de la ville. Les relations à l'œuvre dans cette dynamique, pourtant, ne sont pas aussi simples qu'elles en ont l'air.

\section{Johannesburg, city of quartz}

L'apartheid a disparu pour trois raisons: d'abord, l'arrêt du soutien par les Occidentaux et la condamnation du régime par les Nations Unies, notamment lorsque le Président Reagan rompt avec l'esprit de la Guerre Froide et ne considère plus l'Afrique du Sud comme le dernier bastion du «monde libre» dans une Afrique australe cernée par les communistes. Ensuite, les effets causés dans les années 1980 par les campagnes de défiance vis-à-vis des autorités, prônées par des mouvements de libération comme l'ANC, ont été considérables et ont conduit l'ancien gouvernement à la table des négociations. Enfin, les dynamiques sociales étaient telles qu'un nombre croissant des lois iniques de l'apartheid n'étaient plus respectées, particulièrement dans le domaine du logement et de la relation entre un groupe et son assignation à une aire de résidence bien délimitée (Group Areas Act de 1950, un des piliers de l'apartheid). Entre 1975 et 1995, par exemple, plus de $90 \%$ de la population des quartiers centraux de Johannesburg a changé, alors que le Group Areas Act n'a été aboli qu'en 1991 (Guillaume 1997).

Cette véritable mutation démographique et sociale des espaces centraux de la métropole a été possible grâce à une double dynamique. D'un côté, les vastes appartements du centre de Johannesburg n'ont plus la cote auprès de la classe moyenne et supérieure blanche: les villas avec piscines et jardins des banlieues nord, alors en plein développement, sont beaucoup plus attractives. En même temps, la crise du logement dans les townships bat son plein: le gouvernement 
considère toujours que la citadinité des «non-Blancs» est temporaire. En conséquence, la construction des matchboxes dans les townships est arrêtée au début des années 1970, au profit du développement des bantoustans. Le surplus de logements dans les quartiers «blancs» était estimé à plusieurs milliers en 1985; dans les townships, par contre, il manquait à la même date 5855 logements dans les aires réservées aux Métis, 5135 chez les Indiens et entre 120000 et 140000 chez les Africains. Quelques téméraires se sont donc engouffrés dans la brèche: des familles d'Indiens et de Métis sont venues habiter illégalement vers 1975 dans le centre-ville, grâce à la complaisance de propriétaires soucieux de trouver des locataires. Après quelques péripéties, l'occupation des «non-Blancs» dans ces quartiers est reconnue comme permanente après 1984 (Guillaume 2001; Morris 1999).

À ce moment-là, ces quartiers jouissent d'une image extrêmement positive: ils sont le symbole d'une nouvelle Afrique du Sud, urbaine, mixte, dynamique. Au début des années 1980, Hillbrow est l'emblème de ce renouveau et tire sa réputation de son cosmopolitisme ainsi que par son urbanité renouvelée. Disquaires, librairies, boutiques de mode, cafés, restaurants et boîtes de nuit participent à son rayonnement. Le quartier est vivant, vibrant, coloré, les intellectuels côtoient les artistes et tous ensembles concentrent les espoirs du pays. À la fin des années 1980, il commence toutefois à passer de mode et, en quelques années, le quartier adjacent de Yeoville prend le relais, avec les mêmes arguments. Le rayonnement de Yeoville sera lui aussi de courte durée puisque le quartier perdra une grande partie de son attrait à partir de 1998.

La baisse de l'attractivité de ces quartiers tourne autour d'un seul argument, construit de la manière suivante: Hillbrow et Yeoville ne sont plus ce qu'ils étaient, ils sont beaucoup moins fréquentables qu'auparavant et de plus, très violents. L'alchimie subtile a disparu: cette fusion entre les «races», les classes sociales, les intellectuels, artistes, frimeurs, aventuriers, jolies filles et noctambules a laissé place à un ensemble interlope beaucoup trop voyant, composé de clochards, de gosses de rues, de prostituées, de dealers, de gangs. Ces quartiers ne remplissent plus aucune des règles de la civilité et ne sont plus à même de se maintenir à un minimum acceptable de production culturelle. Même les plus fervents défenseurs de ces lieux finissent par chercher un logement ailleurs et une phase de déclin peut se dérouler complètement en moins de deux ans.

Avec l'évolution de ces quartiers, nous assistons à un glissement de terrain de la violence urbaine: elle opère désormais dans la ville, lieu central et symbo- lique qui structurait fortement, auparavant, la géographie mentale des résidents Blancs de Johannesburg; elle n'est plus réservée à ces endroits obscurs et masqués qu'étaient les townships et les camps de squatters. L'abandon physique du CBD et des quartiers environnants s'est doublé d'une rupture sentimentale: la bourgeoisie (blanche comme noire) s'est reconstruit une nouvelle appartenance à Johannesburg, vingt kilomètres plus au nord dans des quartiers résidentiels dont Sandton est l'emblème. L'urbanisme, depuis peu, illustre clairement la montée des préoccupations sécuritaires. À Sandton et à Randburg il est frappant de noter depuis 1997 la privatisation massive des espaces de vie. Ces quartiers, principalement composés de villas avec piscine, sont de plus en plus regroupés en unités de voisinage, avec des voies d'accès limitées et des postes d'entrée fortement gardés.

Cette fuite en avant se comprend mieux sous l'éclairage particulier de la violence. En Afrique du Sud, selon le Victim \& Crime Survey de 1997, 21\% des foyers ont connu une agression quelconque, 1 foyer sur 200 a déjà été affecté par une affaire de meurtre, 1 personne sur 250 par une affaire d'agression sexuelle ou de viol, et 3,8 millions de Sud-Africains ont déjà été agressés. Ces valeurs, effarantes, concernent le pays tout entier. La province du Gauteng, qui ne couvre même pas $2 \%$ du territoire (mais $18 \%$ de la population du pays - soit 7300000 habitants, urbanisés à $97 \%$ ), se distingue dans presque tous les cas de figure.

Mais avant de passer à l'analyse des tableaux cidessous, il convient de préciser certains éléments. Il est évident que l'on ne peut pas considérer ces données comme fiables, il est préférable de les considérer comme des indications. Par exemple, on estime que $95 \%$ des vols de voiture, $83 \%$ des meurtres, $41 \%$ des cambriolages et $47 \%$ des agressions sexuelles sont signalés à la police. On peut supposer que le taux des incidents signalés va en progressant depuis quelques années, parce que la nature des relations entre la police et les Sud-Africains «non-Blancs» commence à changer, tout doucement. La police, elle même, a dû repenser une grande partie de la nature de ses missions. Cela ne s'est pas fait sans difficultés, compte tenu de l'héritage de l'apartheid et de l'importance de la force dans la gestion des conflits politiques et sociaux, sous l'ancien régime. Gardons également à l'esprit l'héritage de l'apartheid et l'importance de la force dans la gestion des conflits politiques et sociaux, sous l'ancien régime. Il est utile de savoir également que, de 1983 à 1998 , plus de 3000 policiers ont été tués dans l'exercice de leurs fonctions. La violence civile, en Afrique du Sud, n'épargne personne, pas plus les vieillards que les enfants (Tab. 1 et 2). 


\begin{tabular}{|ccc|}
\hline Victimes & $\begin{array}{c}\text { Assassinat avec arme } \\
\text { à feu }\end{array}$ & $\begin{array}{c}\text { Tentative d'assassinat } \\
\text { avec arme à feu }\end{array}$ \\
\hline $0-12$ ans & 791 & 1322 \\
$13-17$ ans & 449 & 825 \\
$18-49$ ans & 8762 & 18912 \\
50 ans et plus & 901 & 1203 \\
Total & $\mathbf{1 0 9 0 3}$ & $\mathbf{2 2} \mathbf{2 6 2}$ \\
\hline
\end{tabular}

Tab. 1: Assassinats et tentatives d'assassinat par armes à feu en Afrique du Sud, 1996

Totschlag und Mordversuche mit Feuerwaffen in Südafrika, 1996

Homicide and attempted murder with firearms in South Africa, 1996

Source: Crime Information Management Centre 1997

L'État sud-africain étant difficilement à même de remplir une de ses missions premières, c'est-à-dire garantir la sécurité de ses citoyens, ce sont les résidents euxmêmes qui, bien souvent, prennent les choses en main. Il est malgré tout évident que l'Etat sud-africain fait de la lutte contre la criminalité une de ses priorités. Le budget du South African Police Service (SAPS) a été augmenté de 6\% de 1998/1999 à 1999/2000, atteignant 15,3 milliards de rands (même valeur en francs français, en l'an 2000), et devrait être de 16,6 milliards de rands en 2001/2002, soit 7\% du budget de l'Etat et $2 \%$ du produit intérieur brut (PIB). 129000 personnes travaillent au SAPS (South African InStITUTE OF Race Relations 2000). Pour l'anecdote, enfin, Johannesburg et Le Cap sont peut-être les seuls endroits au monde où certains commissariats de police font appel à des compagnies privées pour assurer la sécurité de leurs locaux. De nouveaux remparts apparaissent donc: ils ne sont plus lointains comme ceux, symboliques, qui entouraient les townships autrefois, mais leur ombre concrète apaise des citadins qui se sentent assiégés.

De plus en plus de rues sont fermées, temporairement selon les panneaux, et les quartiers doivent présenter çà et là de gigantesques pancartes indiquant le nouveau point d'entrée et de sortie. Il est désormais nécessaire d'intégrer ces nouveaux labyrinthes à ses itinéraires antérieurs, si l'on veut toujours être capable de se déplacer dans Johannesburg. Mais ceci n'est qu'une solution de rattrapage: les villas individuelles sont passées de mode car elles offrent trop peu de sécurité et ce sont maintenant les townhouses, ces complexes de logement collectif à l'intérieur de murs d'enceinte de plusieurs mètres de haut, surmontés de barbelés électrifiés et fermés par des portes blindées, qui font la fortune des promoteurs immobiliers.
Le besoin de sécurité fait partie intégrante du paysage urbain, à l'image de ces plaques armed response qui ornent chaque pan de mur: des animaux agressifs (tigre, pitbull, cobra) sont autant d'exorcismes à l'insécurité, aux hijacks et aux cambriolages. Les compagnies de sécurité privées n'hésitent pas, à l'entrée de certains quartiers ou dans des carrefours stratégiques, à placer des véhicules militaires (souvent des blindés légers) achetés aux domaines et repeints à leurs couleurs. Il s'agit là d'une véritable «militarisation de l'espace urbain", pour reprendre les termes du sociologue américain MiKe Davis (1997), qui illustre à quel point le secteur est florissant.

Entre 1995 et 1997, par exemple, la valeur des compagnies de sécurité privée a augmenté de $167 \%$, pour atteindre plus de 9 milliards de rands (même valeur en francs français en l'an 2000) et près de 6000 compagnies (SCHÖNTEICH 1999), employant plus de 200000 agents de sécurité (LANDMAN 2000); la valeur du marché avait déjà doublé en 1990, au moment de l'abolition de l'apartheid (Tab. 3).

Dans un tel contexte, la progression du nombre des quartiers sécurisés, parfois appelés security villages, fait de cette forme urbaine particulière un élément significatif de la citadinité sud-africaine. Dans ce cas, le quartier, dans sa totalité, résulte d'un projet conduit par un promoteur privé. Il est désormais impossible d'ignorer ces quartiers qui, de plus, ne sont plus seulement réservés à une «élite»: on sait bien que les groupes économiquement les moins favorisés usent aussi de tous les moyens dont ils disposent pour se protéger de l'insécurité. En décembre 1999 et janvier 2000, la sous-structure métropolitaine Nord de Johannesburg a reçu pas moins de 35 demandes de clôture de quartier (LANDMAN 2000). La sous-structure métropolitaine Nord 


\begin{tabular}{|c|c|c|c|c|}
\hline & $\begin{array}{c}\text { Nombre de } \\
\text { cas signalés } \\
1998\end{array}$ & $\begin{array}{c}\text { Nombre de cas / } \\
100000 \\
\text { habitants }\end{array}$ & $\begin{array}{l}\text { Évolution } \\
1994 / 1998\end{array}$ & $\begin{array}{r}\text { Gauteng / } \\
\text { Afrique du } \\
\text { Sud, } 1998\end{array}$ \\
\hline $\begin{array}{l}\text { Violence liée aux taxis } \\
\text { (incidents) }\end{array}$ & 195 & - & $+81 \%$ & $29 \%$ \\
\hline $\begin{array}{l}\text { Violence liée aux taxis } \\
\text { (morts) }\end{array}$ & 111 & - & $+2 \%$ & $38 \%$ \\
\hline Agression & 46922 & 606,5 & $+29 \%$ & $20 \%$ \\
\hline Criminalité liée à la drogue & 5540 & 71,6 & $(+31 \%)$ & $14 \%$ \\
\hline Cambriolage & 96939 & - & $(+0,3 \%)$ & $27 \%$ \\
\hline Possession illégale d'arme & 3269 & 42,3 & $+28,3 \%$ & $22 \%$ \\
\hline Meurtre & 5906 & 76,3 & $(+4,1 \%)$ & $24 \%$ \\
\hline Viol & 11808 & 152,6 & $+9,2 \%$ & $24 \%$ \\
\hline $\begin{array}{c}\text { Agression avec } \\
\text { circonstances aggravantes }\end{array}$ & 41159 & 532 & $+7,8 \%$ & $47 \%$ \\
\hline Vol de voiture & 55031 & 711,3 & $(+2,3 \%)$ & $51 \%$ \\
\hline hijack de voiture & 9213 & 119,1 & $+21 \%$ & $61 \%$ \\
\hline hijack de camion & 3678 & 47,5 & $+61 \%$ & $64 \%$ \\
\hline Attaque de banque & 178 & 2,3 & $(+24,6 \%)$ & $37 \%$ \\
\hline Attaque de transfert de fonds & 53 & 0,7 & $(+38,4 \%)$ & $25 \%$ \\
\hline
\end{tabular}

(Les valeurs entre paranthèses sont des estimations ne tenant pas nécessairement compte du nombre de cas enregistrés.)

Tab. 2: Etat de la violence dans la province du Gauteng, en 1998

Übersicht über Gewaltverbrechen in der Provinz Gauteng, 1998

Overview of acts of violence in Gauteng Province, 1998

Source: South African Institute of Race Relations 2000

de Johannesburg résulte d'un découpage administratif obsolète depuis décembre 2000 , à l'occasion des dernières élections locales. La métropole s'est dotée de l'équivalent d'une cinquième sous-structure et, en même temps, est revenue au concept de megacity et gère tout d'une manière globale, en supprimant un échelon décisionnaire.

Dans un rapport de recherche publié à l'été 2000, Karina Landman fait état d'une étude conduite sous l'égide du Center for Scientific and Industrial Research de Pretoria: un questionnaire portant sur la privatisation des quartiers a été envoyé à 100 autorités locales du pays; 43 ont répondu. La moitié de ces autorités locales soutiennent les idées et projets de privatisation des espaces résidentiels, notamment si cela correspond à une demande des habitants et si cela ne constitue pas une entrave à la circulation. Les opposants font valoir l'idée que les routes et les espaces publics doivent être accessibles à tous, que la gestion des services devient problématique, que la sécurité en cas d'incendie est fortement menacée, que cela gène considérablement les piétons dans la ville et que cela congestionne le trafic automobile ou, plus simplement, que ce n'est pas acceptable dans une démarche globale de gestion des villes. Par ailleurs, ce type de politique ne fait pas forcément l'unanimité au sein des résidents d'un quartier et l'érection d'un mur est un processus binaire dans lequel le consensus ne peut trouver de place. Même si les militants de la fermeture mènent des campagnes un brin démagogiques, distribuent des tracts où l'on doit répondre à un questionnaire avec des questions du type: «Voulez-vous que votre fille soit violée? Voulez-vous pouvoir encore promener votre chien dans la rue?» Si la réponse est, respectivement, non et oui, alors il semble que le mur s'impose comme la seule solution. Nombre de compagnies ont assuré la protection de certains quartiers avec une démarche contraire à la Constitution. Il est vrai qu'il n'existe pas de politique générale concernant la fermeture des quartiers en 


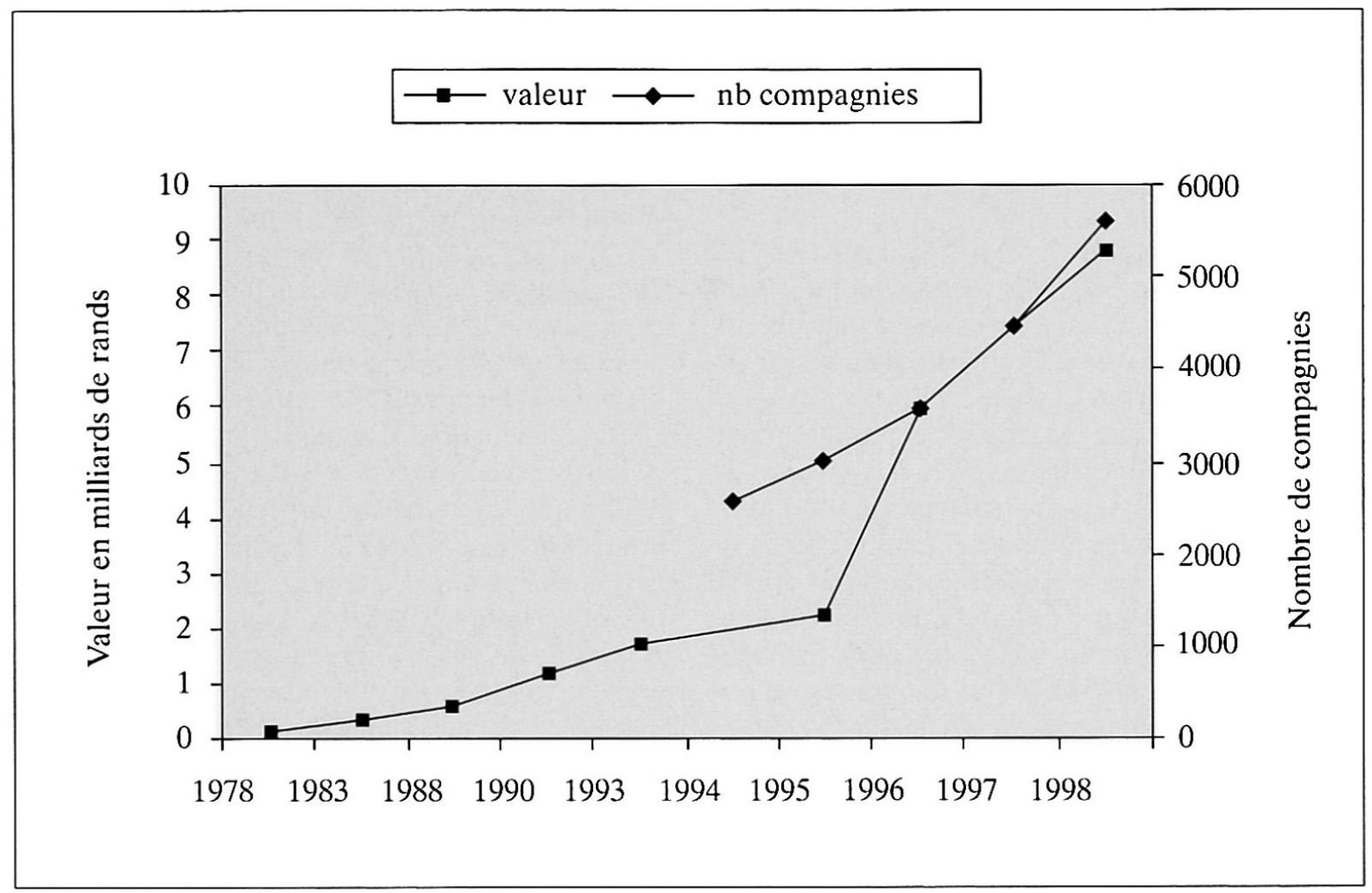

Tab. 3: Evolution du marché de la sécurité privée en Afrique du Sud, 1978-1998

Die Entwicklung des privaten Sicherheitsmarktes in Sïdafrika, 1978-1998

The development of the private security market in South Africa, 1978-1998

Source: d'après SCHÖNTEICH 1999; graphique: L. BAUMANN

Afrique du Sud. La sous-structure métropolitaine Est était l'une des seules autorités locales à avoir exprimé une procédure concernant la privatisation des unités de voisinage: il est par exemple, un peu naïvement écrit que toutes les fermetures de routes devront être temporaires et ne pas excéder douze mois et précise que l'espace public doit rester comme tel. Certaines villes, comme Benoni ou Port-Elizabeth, ont des attitudes beaucoup plus libérales et laissent les résidents "prendre leurs responsabilités».

\section{L'insécurité contre la démocratie ?}

Si les ambiances et les perceptions sont telles que la ville a déjà été présentée par des illustrateurs de presse comme une cité abandonnée en proie aux dinosaures, une lost city en référence au monde perdu de Spielberg, c'est que les faits ne plaident pas en sa faveur. On ne compte plus les articles sur la délinquance et la criminalité, pas plus que ceux qui décrivent la misère des anciens hôtels de luxe devenus des forteresses désertées ni l'irréversible départ des sièges sociaux de bien des compagnies ayant assuré la fortune de l'Afrique du Sud, installées désormais dans les banlieues du nord de la ville. Certains essayistes prédisent l'abandon total des activités lucratives à Johannesburg au profit du Cap. Des oiseaux de mauvais augure, probablement, qui voient un pays en pleine autodestruction.

Métaphore ultime de la déségrégation ratée et foncièrement impossible, le CBD de Johannesburg est affecté de tous les maux. Selon les perceptions populaires, il est non seulement dangereux, mais également composé d'immeubles surpeuplés et en ruine, de quartiers dégradés, d'individus violents, et enfin, d'une absence d'activité économique. La dimension politique de tels propos est fondamentale: parler des quartiers centraux de Johannesburg et de leur déclin permet, sans la nommer tellement la métaphore raciale est évidente, de dire qu'une portion de la société sud-africaine n'est pas mûre pour le pouvoir ni la liberté.

Une analyse plus fine, pourtant, contredit largement ces perceptions. Au début du processus de déségrégation, les propriétaires des immeubles, confrontés à un nombre important d'appartements vide, ont loué plus cher aux nouveaux occupants, ont subdivisé nombre de 
logements pour une rentabilité plus forte et immédiate, et ont par la même occasion cessé d'entretenir les parties communes et l'infrastructure. Les nouveaux arrivants ne pouvaient pas se plaindre, puisqu'ils étaient illégaux.

Dans une étude exhaustive, Alan Morris (1999) a montré que moins de $10 \%$ des logements pouvaient être considérés comme étant surpeuplés; par ailleurs, il a également montré que les immeubles en plus mauvais état, ceux du quartier de Joubert Park, correspondant à la première étape du processus de déségrégation, datent des années 1920, soit vingt à trente ans de plus que ceux des quartiers environnants. Il n'est donc pas étonnant de les voir en mauvais état puisqu'ils ne sont plus entretenus, et le phénomène n'est pas corrélé avec la couleur de peau des habitants. Nous savons également que les nouveaux arrivants de ces quartiers, depuis 1975 , présentent en général des profils éducatifs et socio-économiques plus élevés que les habitants qu'ils remplacent.

Le discours sur le déclin de l'activité économique est également à relativiser. Certes, les plus grandes compagnies quittent le CBD. Cela peut s'interpréter comme une conséquence directe de l'insécurité, mais il est bon de signaler que tous les cadres de ces sociétés habitent dans des quartiers sécurisés du nord de la ville et que venir travailler dans le centre ne les arrange guère. Dire que le $\mathrm{CBD}$ est à l'abandon relève d'une vision centrée sur les seuls Blancs sudafricains: certes, 200000 mètres carrés de bureaux y sont vacants, mais on trouve des immeubles entiers de sociétés gérées par des capitaux «noirs», ce qui n'était pas possible il y a dix ans seulement. De plus, près de 800000 personnes entrent et sortent chaque jour de ce $\mathrm{CBD}$, ce qui ne semble pas cohérent avec la définition d'un espace délaissé; la journée, au moins, le CBD de Johannesburg est extrêmement vivant. Enfin, n'oublions pas que les Sud-Africains «de couleur», auparavant cantonnés dans les townships, disposaient alors d'un espace économique sans grand relief ni potentiel.

Pourtant, on en revient toujours aux faits, à cette violence quotidienne, aveugle et traumatisante. Et il ne sera pas question de nier que, dans toute démocratie, la sécurité est une exigence primordiale pour les citoyens. En Afrique du Sud, justement, le passage à la démocratie a fait baisser les perceptions des sentiments de sécurité: en 1994, 48\% des citoyens pensaient avoir un contrôle sur leur sécurité; $63 \%$ pensaient le contraire en 1997 (SCHÖNTEICH 1999). Cela conduit, en toute logique, à la valorisation des espaces privés: dans une enquête, $79 \%$ des femmes se sentent en insécurité dans les espaces publics, $21 \%$ au contraire se sentent plus menacées dans les espaces privés; par ailleurs, $65 \%$ des sondés se considèrent en insécurité la nuit dans leur quartier de résidence à Johannesburg, contre $50 \%$ à Pretoria et $41 \%$ au Cap (Landman 2000). Avec, en filigrane, ce discours muet: la «libération» des Africains a conduit au chaos et au déclin du pays.

Tenir compte de ces données brutes, sans les remettre en question, ce serait oublier que le rapport des hommes au paysage est empli de symboles. Aussi, dès les premiers signes d'évolution d'un quartier, les boucliers se lèvent et les discours changent instantanément. À Bertrams, à l'est du CBD, l'implantation d'enseignes de banques portugaises, de dentistes en zoulou et de boucherie halaal a été vécue comme une agression dans ce quartier jusque là composé d'Afrikaners vivant dans des logements municipaux. Dans une enquête menée à Yeoville en 1988, il apparaissait que la déségrégation était perçue comme une menace. Les résidents s'inquiétaient de la montée de l'alcoolisme, de l'établissement de shebeens, du vagabondage dans les lieux publics. Dès 1983, des journaux populaires tels que THE Citızen rapportaient l'augmentation de la criminalité, que les «gens de couleur» dégradaient le quartier, urinaient en public et «que le mode de vie traditionnel des Blancs était menacé». À l'époque, le quartier est pourtant «libéral», peuplé de journalistes, étudiants, universitaires, artistes. Cela ne permet pourtant pas d'éviter les fantasmes et la mythologie: si les premiers habitants «non-Blancs» sont arrivés à Yeoville en 1985, c'est en 1991 que le processus de déségrégation a été véritablement sensible, et encore, sans modifier totalement l'allure du quartier: de 1991 à 1995, les Noirs et Indiens ne sont entrés que dans $9,3 \%$ des transactions immobilières, portant sur 323 maisons et appartements (BÄHR, BocK \& JÜRGENS 1998).

Ce type de phénomène n'est pas spécifique à Johannesburg: lors de l'établissement de nouveaux arrivants dans des quartiers du Cap ou de Durban en 1990, la population blanche s'est immédiatement et systématiquement plainte d'une augmentation de la violence et de la criminalité. La police, évidemment, ne corroborait pas ces perceptions.

Permettons nous quelques déductions, à la lumière de ces éléments. Il est évident que la société blanche sudafricaine n'était pas prête à l'arrivée de groupes «d'intrus» dans des espaces qu'elle s'était réservés. Dès lors, chaque marque d'installation de ces "Autres», pour légitime, naïve ou anecdotique qu'elle fût, a été vécue comme une agression. Ce sentiment ne permet pas de partager l'espace, de créer ensemble de nouvelles pratiques ou de nouveaux paysages. De fait, par des processus sociaux semblables aux prophéties auto-réalisatrices (StaszaK 2000), ce qui était craint s'est bien produit. 
L'insécurité joue donc bien contre la démocratie, mais pas plus par sa concrétisation quotidienne que par sa position de force dans un discours justifiant l'absence d'ouverture, de partage, de fusion.

\section{Conclusion}

En sept ans, les forces qui modèlent la société urbaine sud-africaine ont bien changé et le fossé se creuse entre les orientations politiques et les dynamiques sociales. Les autorités locales souhaitent toujours gouverner en tenant compte des besoins de tous les citoyens, en ayant une vision à long terme et en privilégiant les espaces publics. Le pouvoir central semble toujours y croire, comme en témoigne le Development Facilitation Act de 1999 qui veut donner des atouts à une gestion des villes privilégiant la densité et le contact, le tout dans une approche holistique. De l'autre côté, l'explosion du discours sécuritaire conduisant à une privatisation croissante des espaces, modifiant les définitions des communautés, conduisant à un déplacement et non à une éradication de la violence, posant des problèmes quotidiens de circulation et de services et, surtout, posant des interrogations à long terme sur l'avenir et la démocratie des villes. Et, entre les deux, $50 \%$ de la population du pays vivant en dessous du seuil de pauvreté et se partageant $11 \%$ des ressources.

Concluons cet essai par deux tableaux. À Kliptown, dans un bidonville du township de Soweto, se trouve une fresque sur le bureau du club de la jeunesse, animé par des bénévoles souhaitant détourner enfants et adolescents de la violence et de la criminalité. On y trouve des messages d'amour, de tolérance et de fraternité, de respect et d'espoir qui jouxtent des slogans prévenant contre les armes, la drogue ou le SIDA. À Sandton City, dans le plus grand et plus luxueux shopping mall, une fresque se trouve dans un passage en trompe l'œil, donnant au piéton l'impression de marché dans une rue aux murs pastels. De l'autre côté des murs: des bureaux et le parking souterrain. Sur la fresque, la place SaintMarc. Réinvention de l'agora dans la caricature de l'espace privé, clinquant et commerçant. Cette société a vraiment besoin d'une réflexion collective.

\section{Bibliographie}

BäHR, J., Воск, S. \& U. Jürgens (1998): Auflösung der Segregation in der Post-Apartheid-Stadt? Diskutiert anhand kleinräumiger Wohnungsmarktanalysen im Großraum Johannesburg. - = Petermanns Geographische Mitteilungen 142, 1:3-18.

BrinK, A. (1979): Rumeurs de pluie. - Paris: Stock. Coetzee, M. (1992): L'Age de Fer. - Paris: Seuil.

Davis, M. (1997): City of Quartz. Los Angeles, capitale du futur. - Paris: La Découverte.
Foucault, M. (1975): Surveiller et punir: naissance de la prison. - Paris: Gallimard.

GLaSER, C. (1988): We must infiltrate the tsotsis: school politics and youth gangs in Soweto, 1968-1976. - In: Journal of Southern African Studies 24, 2: 301-323.

Guillaume, P. (1997): Du blanc au noir... Essai sur une nouvelle ségrégation dans le centre de Johannesburg. - In: L'Espace Géographique 26, 1:21-33.

Guillaume, P. (2001): Johannesburg. Géographies de l'exclusion. - Paris/Johannesburg: Karthala/IFAS.

LAINÉ, D. (1999): Johannesburg. Un mauvais rêve. - In: Géo 242: 56-72.

LANDMAN, K. (2000): An overview of enclosed neighbourhoods in South Africa. - Pretoria: CSIR.

LÉvy, J. (1999): Le tournant géographique. Penser l'espace pour lire le monde. - Paris: Belin.

DE Montclos, M.-A. (1997): Violence et sécurité urbaine en Afrique du Sud et au Nigeria. Un essai de privatisation. -Paris: L'Harmattan, 2 tomes.

Morris, A. (1999): Bleakness \& light. Inner-city transition in Hillbrow, Johannesburg. - Johannesburg: Witwatersrand University Press.

SCHÖNTEICH, M. (1999): Unshackling the crime fighters. Increasing private sector involvment in South Africa's criminal justice system. - Johannesburg: South African Institute of Race Relations.

South African Institute of Race Relations (2000): South African Survey, 1999-2000. - Johannesburg (http://www.sairr.org.za).

Staszak, J.-F. (2000): Prophéties autoréalisatrices et géographie. - In: L'Espace Géographique 29, 2: 105-119.

TRÉTIACK, P. (1999): Johannesburg, radieux coupegorge. - In: Le Monde des Débats 3:36-37.

\section{Résumé: La violence urbaine à Johannesburg. Entre réalité et prétexte}

S'affranchir de l'incompréhension, du racisme, de l'intolérance, reconstruire des institutions, repenser les relations entre les groupes, combler le gouffre, réinventer la démocratie - voilà des objectifs parmi les plus beaux que peut porter notre monde d'un nouveau millénaire. Ils sont à l'œuvre en Afrique du Sud, mais risquent de buter sur un acteur sournois et d'une préhension difficile: la violence. Il convient alors de distinguer la part réelle de la violence de ces sociétés, inadmissible, de son rôle dans les discours et de ses conséquences dans les dynamiques sociales. Et si la violence pouvait agir comme prétexte et servir les intérêts de ceux qui s'en prétendent les victimes?

\section{Abstract: Violent Urban Crime in Johannesburg. Between Reality and Pretence}

To reduce intolerance, racism, misunderstanding, to build institutions, to rethink social relations, to close development differentials, to redefine democracy - 
those are some of the great challenges for the $21^{\text {st }}$ century. Major efforts are being made in South Africa but they encounter a malicious enemy: violence. Violence needs to be studied in terms of the role it plays in disrupting society, in the discourse on the fabric of society and the dynamics of societal development. Attention must also be given to the question whether or not violence serves as a pretext for the special interests of those who pretend to be victims. The aim of this article is to discuss these issues in the context of Johannesburg, South Africa.

\section{Zusammenfassung: Die städtische Gewalt in Johannesburg. Zwischen Realität und Vorwand} Wegkommen von Intoleranz, Rassismus und Missverständnis, Institutionen einrichten, soziale Beziehungen überdenken, den Graben schliessen, die Demokratie neu erfinden - dies sind einige der grossen Herausforderungen des 21. Jahrhunderts. Diesbezügliche Bemühungen sind in Südafrika im Gang, doch sie können über einen heimtückischen Akteur stolpern: die Gewalt. Wir sollten darum den wirklichen Part der Gewalt in der Gesellschaft untersuchen und seine Rolle in den allgemeinen Diskursen und seine Folgen auf die soziale Dynamik unterscheiden. Der Aufsatz behandelt diesen Diskurs, auch die Frage, ob die Gewalt als ein Vorwand und den Interessen derer dient, die behaupten, die Opfer zu sein.

\section{Orientations didactiques}

- Quelles sont les causes sociales de la violence dans la ville de Johannesburg?

- Comment vivent les habitants des townships?

- Qu'est-ce qui différencie principalement la vie des Blancs avant et après la fin du régime de l'apartheid?

- Comment les quartiers centraux de Johannesbourg se sont-ils développés après 1980 ?

- De quelle(s) manière(s) les citadins réagissent-ils face à la progression de la violence urbaine en Afrique du Sud?

- Quelles sont les principales différences entre ce que l'on a appelé d'une manière générale le processus de ségrégation dans les villes nord-américaines et le processus sud-africain d'apartheid?

- Quelle est la nature des relations socio-spatiales au sein des townships d'Afrique du Sud?

Dr. Philippe Guillaume, 15, avenue du 25 août 1944, F-94600 Choisy-le-Roi.

e-mail: philippe_guillaume@ananzi.co.za

\section{Manuskripteingang/received/manuscrit entré le} 21.1.2004

Annahme zum Druck/accepted for publication/accepté pour l'impression: 20.8.2004 